\title{
The Subgroup Theorem
}

\author{
By
}

ROBERT KUBOTA

We give a simplified proof of a theorem of Federer and Jónsson [1], which contains NIELSEN's theorem, that every subgroup of a free group is free.

Theorem. Let $F$ be a free group with basis $X$, and, for $a \in F$, let $|a|$ be the length of $a$ relative to $X$. Let $G$ be a subgroup of $F$, well ordered by a relation $\prec$ such that $|a|<|b|$ implies $a<b$. For $a \in G$, let $G_{a}=\operatorname{gp}\{b \in G \mid b \prec a\}$. Let $A=\left\{a \in G \mid a \notin G_{a}\right\}$. Then $G$ is free on the basis $A$.

We prove that $A$ generates $G$. Suppose not. Let $c$ be the least member of $G-\operatorname{gp} A$. Then $c \notin A$, whence $c \in G_{c}$. But $G_{c}$ is generated by elements $b \prec c$ which, by the minimality of $c$, lie in $\mathrm{gp} A$, whence $G_{c} \subseteq \mathrm{gp} A$. This implies that $c \in \mathrm{gp} A$, a contradiction.

We prove that $A$ is a free basis for $G$. Suppose not. Then there is a relation $u_{1} \cdots u_{n}=1$ where $n \geqq 1, u_{i}^{ \pm 1} \in A$, and $u_{i} \neq u_{i+1}^{-1}$. Let $a$ be maximal among the $u_{i}^{+1} \in A$. We may suppose our relation chosen such that $a$ is minimal, and hence such that $A_{a}=\{b \in A \mid b \prec a\}$ is a basis for a free group. Note that, for $1 \leqq i \leqq n$, either $u_{i}=a^{ \pm 1}$ or $u_{i} \in G_{a}$, and in either case $\left|u_{i}\right| \leqq|a|$.

The sequence $u_{1}, \ldots, u_{n}$ satisfies the following conditions:

$$
\begin{aligned}
& \text { For } 1 \leqq i \leqq n-1, \quad u_{i} \neq u_{i+1}^{-1} . \\
& \text { For } 1 \leqq i \leqq n, \quad 0<\left|u_{i}\right| \leqq|a| \\
& \text { For } \quad 1 \leqq i \leqq n, \text { either } u_{i} \in G_{a} \text { or } u_{i}=a^{ \pm 1} . \\
& \text { For some } i, 1 \leqq i \leqq n, \quad u_{i}=a^{ \pm 1} . \\
& u_{1} \cdots u_{n}=1
\end{aligned}
$$

If, for some $i, 1 \leqq i \leqq n-1$, we have $u_{i}, u_{i+1} \in G_{a}$ and $\left|u_{i} u_{i+1}\right| \leqq|a|$, then we replace the two factors $u_{i}$ and $u_{i+1}$ by a single factor $u_{i} u_{i+1}$ to obtain a sequence $v_{1}, \ldots, v_{n-1}$ of $n-1$ factors. Since $A_{a}$ is a basis for a free group not containing $a$, the sequence $v_{1}, \ldots, v_{n-1}$ satisfies $(1)-(6)$, and iteration of this process will yield a new sequence satisfying the further condition:

$$
\text { For } 1 \leqq i \leqq n-1 \text {, if } u_{i}, u_{i+1} \in G_{a} \text { then }\left|u_{i} u_{i+1}\right|>|a|
$$

Lemma 1. If $1 \leqq i \leqq n-1$, then $\left|u_{i} u_{i+1}\right| \geqq\left|u_{i}\right|,\left|u_{i+1}\right|$, and both inequalities are strict unless exactly one of $u_{i}, u_{i+1}$ is $a^{ \pm 1}$. 
Proof. Either $u_{i} \in G_{a}$ or $u_{i}=a^{ \pm 1}$, and either $u_{i+1} \in G_{a}$ or $u_{i+1}=a^{ \pm 1}$. The case that $u_{i}, u_{i+1} \in G_{a}$ is given by (7). The case that $u_{i}=u_{i+1}^{-1}=a^{ \pm 1}$ is excluded by (1). In the case that $u_{i}=u_{i+1}=a^{ \pm 1}$, since $a \neq 1$ we have $\left|a^{ \pm 2}\right|>|a|$. The case remains that one of $u_{i}, u_{i+1}$ is in $G_{a}$ and the other is $a^{ \pm 1}$. Suppose $u_{i} \in G_{a}$ and $u_{i+1}=a^{ \pm 1}$. Then $\left|u_{i} u_{i+1}\right|<|a|$ would imply $u_{i} u_{i+1} \in G_{a}$. This, with $u_{i} \in G_{a}$ would give $a=$ $=u_{i+1}^{ \pm 1} \in G_{a}$, contrary to $a \in A$.

Lemma 2. If $2 \leqq i \leqq n-1$ and $u_{i}$ cancels exactly half in each of its neighbors, that is,

then either:

$$
\left|u_{i-1} u_{i}\right|=\left|u_{i-1}\right| \text { and }\left|u_{i} u_{i+1}\right|=\left|u_{i+1}\right|
$$

$$
u_{i}=a^{ \pm 1} ; \quad u_{i-1}, u_{i+1} \neq a^{ \pm 1} ; \quad\left|u_{i-1}\right|=\left|u_{i+1}\right|=|a| ;
$$

and exactly half of each of $u_{i}, u_{i+1}$ remains in $u_{i-1} u_{i} u_{i+1}$; or:

$$
u_{i-1}=u_{i+1}=a^{ \pm 1} ; \quad\left|u_{i}\right|<|a| ;
$$

and more than half of each of $u_{i-1}, u_{i+1}$ remains in $u_{i-1} u_{i} u_{i+1}$.

Proof. By Lemma 1, we must have either (A): $u_{i}=a^{ \pm 1}$ and $u_{i-1}, u_{i+1} \in G_{a}$; or (B): $u_{i} \in G_{a}$ and $u_{i-1}, u_{i+1}=a^{ \pm 1}$.

In Case A, Lemma 1 gives $|a|=\left|u_{i}\right| \leqq\left|u_{i-1} u_{i}\right|=\left|u_{i-1}\right|$, whence, by (2), $\left|u_{i-1}\right|=|a|$; similarly, $\left|u_{i+1}\right|=|a|$. If there were cancellation between $u_{i-1}$ and $u_{i+1}$ we should have $\left|u_{i-1} u_{i} u_{i+1}\right|<|a|$ and so $u_{i-1} u_{i} u_{i+1} \in G_{a}$. This with $u_{i-1}$, $u_{i+1} \in G_{a}$ would give $a=u_{i}^{ \pm 1} \in G_{a}$, contrary to $a \in A$.

In Case $\mathrm{B}$, we can write $u_{i}=p q$ where $|p|=|q|=\frac{1}{2} \cdot\left|u_{i}\right|$. Now $u_{i-1}=u_{i+1}^{-1}=$ $=a^{ \pm 1}$ would imply that $p=q^{-1}$ and $u_{i}=1$, contrary to (2). Therefore $u_{i-1}=$ $=u_{i+1}=a^{ \pm 1}$ and we can write $a^{ \pm 1}=q^{-1} r p^{-1}$ for some $r$. Now $\left|u_{i}\right|=|a|$ would imply that $r=1$ and $u_{i}=a \pm 1$, contrary to (1). Therefore $\left|u_{i}\right|<|a|$ and $r \neq 1$. If as much as half of one of $u_{i-1}$ or $u_{i+1}$, and so of both, cancelled in the product $u_{i-1} u_{i} u_{i+1}=q^{-1} r^{2} p^{-1}$, then each factor $r$ in $r^{2}$ would have to cancel at least half, giving $\left|r^{2}\right| \leqq|r|$, which is not possible for $r \neq 1$.

Since, by (5), $u_{1} \cdots u_{n}=1$, the proof of the theorem will be complete when we have established the following lemma.

Lemma 3, $0<\left|u_{1}\right| \leqq\left|u_{1} u_{2}\right| \leqq \cdots \leqq\left|u_{1} \cdots u_{n}\right|$.

Proof. We write $p_{i}=u_{1} \cdots u_{i}$. We shall show, by induction on $i$, that $0<\left|p_{1}\right| \leqq$ $\leqq \cdots \leqq\left|p_{i}\right|$ and that $\left|p_{i-2}\right|=\left|p_{i-1}\right|=\left|p_{i}\right|$ only in case that $\left|p_{i-3}\right|<\left|p_{i-2}\right|$ and that $u_{i-2}, u_{i-1}, u_{i}$ fall under Case A of Lemma 2 . For $i=1,2,3$, this follows directly from (2) and Lemmas 1 and 2 . We assume this condition for some $i, 3 \leqq i \leqq n-1$, and shall prove it for $i+1$.

Suppose $\left|p_{i-1}\right|<\left|p_{i}\right|$; then we must show that $\left|p_{i}\right| \leqq\left|p_{i+1}\right|$. Now $\left|p_{i-1}\right|<\left|p_{i}\right|$ implies that more than half of $u_{i}$ remains in $p_{i}$; since at most half of $u_{i}$ cancels in $u_{i} u_{i+1}$, some part of $u_{i}$ remains in $p_{i+1}$. Therefore as much of $u_{i+1}$ remains in $p_{i+1}$ as in $u_{i} u_{i+1}$, that is, at least half, and $\left|p_{i}\right| \leqq\left|p_{i+1}\right|$. 
Suppose $\left|p_{i-2}\right|<\left|p_{i-1}\right|=\left|p_{i}\right|$; we must show that $\left|p_{i}\right| \leqq\left|p_{i+1}\right|$, with equality only under Case A. Now part of $u_{i-1}$ remains in $p_{i}$, whence as much of $u_{i+1}$ remains in $p_{i+1}$ as in $u_{i-1} u_{i} u_{i+1}$, and the conclusion follows by Lemma 2 .

Finally, suppose that $\left|p_{i-2}\right|=\left|p_{i-1}\right|=\left|p_{i}\right|$; we must show that $\left|p_{i}\right|<\left|p_{i+1}\right|$. By the induction hypothesis, $u_{i-2}, u_{i-1}, u_{i}$ fall under Case $\mathrm{A}$, and half of $u_{i}$ remains in $p_{i}$. Then $\left|u_{i}\right|=|a|$, and $u_{i-1}, u_{i}, u_{i+1}$ cannot fall under Lemma 2 , whence less than half of $u_{i}$ cancels in $u_{i} u_{i+1}$, and part of $u_{i}$ remains in $p_{i+1}$. But then $u_{i+1}$ cancels no more in $p_{i+1}$ than in $u_{i} u_{i+1}$. If $u_{i+1}=a^{ \pm 1}$ this is less than $u_{i}$ cancels in $u_{i} u_{i+1}$, hence less than half, while if $u_{i+1} \in G_{a}$, this is less than half by (7). In both cases, $\left|p_{i}\right|<$ $<\left|p_{i+1}\right|$.

\section{Referenee}

[1] H. Federer and B. Jónsson, Some properties of free groups. Trans. Amer. Math. Soc. 68, $1-27(1950)$.

Eingegangen am 12.5.1964

Anschrift des Autors:

Robert Kubota

c.o. R. C. Lyndon

Mathematics Angell Hall

University of Michigan

Ann Arbor (Mich.), USA 